\title{
A BLICHFELDT-TYPE INEQUALITY FOR CENTRALLY SYMMETRIC CONVEX BODIES
}

\author{
MATTHIAS HENZE
}

\begin{abstract}
In this note, we derive an asymptotically sharp upper bound on the number of lattice points in terms of the volume of centrally symmetric convex bodies. Our main tool is a generalization of a result of Davenport that bounds the number of lattice points in terms of volumes of suitable projections.
\end{abstract}

\section{INTRODUCTION}

Let $\mathcal{K}^{n}$ be the set of all convex bodies in $\mathbb{R}^{n}$, i.e., compact convex sets $K$ with nonempty interior int $K$. Such a body $K$ is called centrally symmetric if $K=-K$. The family of $n$-dimensional lattices in $\mathbb{R}^{n}$ is denoted by $\mathcal{L}^{n}$ and the usual Lebesgue measure with respect to the $n$-dimensional Euclidean space by $\operatorname{vol}_{n}(\cdot)$. If the ambient space is clear from the context, we omit the subscript and just write $\operatorname{vol}(\cdot)$. For a bounded subset $S \subset \mathbb{R}^{n}$ the lattice point enumerator is denoted by $\mathrm{G}(S)=\#\left(S \cap \mathbb{Z}^{n}\right)$. A lattice polytope is a polytope all of whose vertices are lattice points in $\mathbb{Z}^{n}$. Finally, for an $A \subseteq \mathbb{R}^{n}$ we denote the dimension of its affine hull by $\operatorname{dim} A$.

We are interested in bounds on the volume in terms of the lattice point enumerator of a convex body. For $K \in \mathcal{K}^{n}$ with $\operatorname{dim}\left(K \cap \mathbb{Z}^{n}\right)=n$ a sharp lower bound on $\operatorname{vol}(K)$ was obtained by Blichfeldt [4], which reads

$$
\operatorname{vol}(K) \geq \frac{1}{n !}(\mathrm{G}(K)-n) .
$$

We call results of this kind Blichfeldt-type inequalities. On the other hand, the best known upper bound on $\operatorname{vol}(P)$ for a lattice polytope $P \in \mathcal{K}^{n}$ is due to Pikhurko [15]

$$
\operatorname{vol}(P) \leq(8 n)^{n} 15^{n 2^{2 n+1}} \mathrm{G}(\text { int } P)
$$

and holds under the condition that $\mathrm{G}(\operatorname{int} P) \neq 0$. On the class of centrally symmetric convex bodies, Blichfeldt [4] and van der Corput [6] obtained a sharp upper bound on the volume

$$
\operatorname{vol}(K) \leq 2^{n-1}(\mathrm{G}(\operatorname{int} K)+1) \quad \text { for all } \quad K \in \mathcal{K}_{0}^{n} .
$$

2010 Mathematics Subject Classification. 52C07, 52B20, 52A40, $11 \mathrm{H} 06$.

Key words and phrases. Blichfeldt-type inequalities, Davenport inequality, central symmetry, lattice point enumerator.

This work was supported by the Deutsche Forschungsgemeinschaft (DFG) within the project He 2272/4-1. 
Bey, Henk and Wills [3] proposed the study of a reverse inequality also on the class of centrally symmetric convex bodies, and in [11] the authors derive, as a first step, Blichfeldt-type inequalities for lattice crosspolytopes, lattice zonotopes, and for centrally symmetric planar convex sets. Moreover, they conjecture that there is a constant $c>1$ such that $\operatorname{vol}(K) \geq \frac{c^{n}}{n !} \mathrm{G}(K)$ for every $K \in \mathcal{K}_{0}^{n}$ with $\operatorname{dim}\left(K \cap \mathbb{Z}^{n}\right)=n$.

In this work, we confirm this conjecture asymptotically by showing that for every $\varepsilon \in(0,1]$ and large enough $n \in \mathbb{N}$ a valid choice for this constant is $c=2-\varepsilon$. As the main ingredient to our argument, we prove the following generalization of a classical result of Davenport [7]. Therein, we denote by $\mathcal{Q}(P)$ the set of all lattice parallelepipeds in $\mathcal{K}^{n}$ whose edges are parallel to a given lattice parallelepiped $P \in \mathcal{K}^{n}$. Moreover, $K \mid L$ denotes the orthogonal projection of $K$ onto the subspace $L$ and $\left(\begin{array}{c}{[n]} \\ i\end{array}\right)$ is the set of all $i$-element subsets of $[n]=\{1, \ldots, n\}$.

Lemma 1.1. Let $K \in \mathcal{K}^{n}$ and let $P=\sum_{j=1}^{n}\left[0, z_{j}\right]$ be a lattice parallelepiped. Then

$$
\mathrm{G}(K) \leq \sum_{i=0}^{n} \sum_{J \in\left(\begin{array}{c}
{[n]} \\
i
\end{array}\right)} \operatorname{vol}_{n-i}\left(K \mid L_{J}^{\perp}\right) \operatorname{vol}_{i}\left(P_{J}\right),
$$

where $L_{J}=\operatorname{lin}\left\{z_{j}: j \in J\right\}$ and $P_{J}=\sum_{j \in J}\left[0, z_{j}\right]$ for each $J \in\left(\begin{array}{c}{[n]} \\ i\end{array}\right)$. Equality holds if and only if $P$ is a fundamental cell of $\mathbb{Z}^{n}$ and $K \in \mathcal{Q}(P)$.

As mentioned above, we use the preceding result to derive a Blichfeldttype inequality for centrally symmetric convex bodies and thereby confirm conjectured bounds from [3, Conj. 1.1] and [11] asymptotically.

Theorem 1.1. For every $\varepsilon \in(0,1]$ there exists an $n(\varepsilon) \in \mathbb{N}$ such that for every $n \geq n(\varepsilon)$ and every $K \in \mathcal{K}_{0}^{n}$ with $\operatorname{dim}\left(K \cap \mathbb{Z}^{n}\right)=n$, we have

$$
\operatorname{vol}(K) \geq \frac{(2-\varepsilon)^{n}}{n !} \mathrm{G}(K)
$$

The constant 2 cannot be replaced by a bigger one.

As an application of this inequality we bound the magnitude $\frac{\mathrm{G}(K)}{\mathrm{G}\left(K^{\star}\right) \operatorname{vol}(K)}$ by constants that depend on the dimension $n$ but not on the body $K$. Recall that $K^{\star}=\left\{x \in \mathbb{R}^{n}: x^{\top} y \leq 1, \forall y \in K\right\}$ denotes the polar body of $K \in \mathcal{K}_{0}^{n}$. Estimates of such kind were first studied and applied by Gillet and Soulé [9] who obtained $6^{-n} \leq \frac{\mathrm{G}(K)}{\mathrm{G}\left(K^{\star}\right) \operatorname{vol}(K)} \leq \frac{6^{n} n !}{c^{n}}$ for some absolute constant $c \leq 4$.

Theorem 1.2. For every $\varepsilon>0$ there exists an $n(\varepsilon) \in \mathbb{N}$ such that for every $n \geq n(\varepsilon)$ and every $K \in \mathcal{K}_{0}^{n}$ with $\operatorname{dim}\left(K \cap \mathbb{Z}^{n}\right)=n$, we have

$$
(\pi+\varepsilon)^{-n} \leq \frac{\mathrm{G}(K)}{\mathrm{G}\left(K^{\star}\right) \operatorname{vol}(K)} \leq \frac{(\pi+\varepsilon)^{n} n !}{c^{n}},
$$

where $c \leq 4$ is an absolute constant.

The results of Lemma 1.1 and Theorem 1.1 are discussed in the subsequent section. In Section 3, we give the details of the proof of Theorem 1.2. 


\section{Proofs of Lemma 1.1 And Theorem 1.1}

A convex body $T \in \mathcal{K}^{n}$ is said to be a lattice tile with respect to the lattice $\Lambda \in \mathcal{L}^{n}$ if $T$ tiles $\mathbb{R}^{n}$ by vectors in $\Lambda$, that is, $\mathbb{R}^{n}=\Lambda+T$ and $(x+\operatorname{int} T) \cap(y+\operatorname{int} T)=\emptyset$, for all different $x, y \in \Lambda$. It is well-known that lattice tiles are polytopes, and thus we can assume that every lattice tile has the origin as a vertex. For a survey on tilings and references to the relevant literature, we refer the reader to [19].

Betke and Wills [1] (cf. [10, Sect. 3]) showed that, for every convex body $K \in \mathcal{K}^{n}$, the number of lattice points in $K$ is bounded by $\mathrm{G}(K) \leq \operatorname{vol}(K+L)$, where $L$ is a fundamental cell of $\mathbb{Z}^{n}$. They asked to determine all bodies $L$ that admit such an inequality. With the following lemma, we identify lattice tiles as bodies with this property.

Lemma 2.1. Let $K \in \mathcal{K}^{n}$ and let $T$ be a lattice tile with respect to a sublattice $\Lambda$ of $\mathbb{Z}^{n}$. Then

$$
\mathrm{G}(K) \leq \operatorname{vol}(K+T) .
$$

If $T$ is a lattice parallelepiped $P$, then equality holds if and only if $\Lambda=\mathbb{Z}^{n}$ and $K \in \mathcal{Q}(P)$.

Proof. Since for all $x, y \in \Lambda$ we have $(x+\operatorname{int} T) \cap(y+\operatorname{int} T)=\emptyset$, unless $x=y$, every residue class modulo $\Lambda$ is a packing set of $T$. Let $\left\{r_{1}, \ldots, r_{m}\right\} \subset \mathbb{Z}^{n}$ be a maximal subset of different representatives of residue classes modulo $\Lambda$. Writing $\Lambda_{j}=r_{j}+\Lambda$, we have for every $j=1, \ldots, m$ that

$$
\#\left(K \cap \Lambda_{j}\right)=\frac{\operatorname{vol}\left(\left(K \cap \Lambda_{j}\right)+T\right)}{\operatorname{vol}(T)} .
$$

Since $T$ is a lattice tile, we have $\operatorname{vol}(T)=\operatorname{det} \Lambda=m$, and therefore

$$
\mathrm{G}(K)=\sum_{j=1}^{m} \#\left(K \cap \Lambda_{j}\right)=\frac{1}{m} \sum_{j=1}^{m} \operatorname{vol}\left(\left(K \cap \Lambda_{j}\right)+T\right) \leq \operatorname{vol}(K+T) .
$$

By the compactness of the involved sets, equality is attained if and only if $\left(K \cap \Lambda_{j}\right)+T=K+T$ for all $j=1, \ldots, m$. In particular, there can only be one residue class and thus $m=\operatorname{det} \Lambda=1$, which means $\Lambda=\mathbb{Z}^{n}$. In the case that the lattice tile is a lattice parallelepiped $P=\sum_{i=1}^{n}\left[0, a_{i}\right]$, every hyperplane supporting a facet of the convex polytope $K+P=\left(K \cap \mathbb{Z}^{n}\right)+P$ is parallel to a hyperplane supporting a facet of $P$. Therefore, $K+P$ is a lattice translate of $\sum_{i=1}^{n}\left[0, t_{i} a_{i}\right]$ for some $t_{i} \in \mathbb{N}$, and so $K$ is a lattice translate of $\sum_{i=1}^{n}\left[0,\left(t_{i}-1\right) a_{i}\right] \in \mathcal{Q}(P)$.

Conversely, if $P$ is a fundamental cell of $\mathbb{Z}^{n}$, then we find lattice vectors $v_{1}, \ldots, v_{n} \in \mathbb{Z}^{n}$ such that, up to a lattice translation, $P=\sum_{i=1}^{n}\left[0, v_{i}\right]$. Again, up to a lattice translation, every $K \in \mathcal{Q}(P)$ is of the form $K=$ $\sum_{i=1}^{n}\left[0, l_{i} v_{i}\right]$ for some $l_{1}, \ldots, l_{n} \in \mathbb{N}$. Since $P$ is a fundamental cell, we have 


$$
\begin{aligned}
\operatorname{vol}(P)=1= & \#\left(\sum_{i=1}^{n}\left[0, v_{i}\right) \cap \mathbb{Z}^{n}\right) \text { and thus } \\
& \mathrm{G}(K)=\#\left(\sum_{i=1}^{n}\left[0,\left(l_{i}+1\right) v_{i}\right) \cap \mathbb{Z}^{n}\right)=\operatorname{vol}(K+P) .
\end{aligned}
$$

Proof of Lemma 1.1. The lattice parallelepiped $P=\sum_{j=1}^{n}\left[0, z_{j}\right]$ is clearly a lattice tile with respect to the sublattice of $\mathbb{Z}^{n}$ which is spanned by $z_{1}, \ldots, z_{n}$. Based on an alternative proof by Ulrich Betke of an inequality of Davenport [7] we use Lemma 2.1 and develop the volume of $K+P$ into a sum of the mixed volumes $\mathrm{V}(K, n-i ; P, i)$ of $K$ and $P$ (we refer to the books of Gardner [8] and Schneider [18] for details and properties on mixed volumes)

$$
\mathrm{G}(K) \leq \operatorname{vol}(K+P)=\sum_{i=0}^{n}\left(\begin{array}{c}
n \\
i
\end{array}\right) \mathrm{V}(K, n-i ; P, i) .
$$

By the linearity and nonnegativity of the mixed volumes we have

$$
\begin{aligned}
\mathrm{V}(K, n-i ; P, i) & =\sum_{j_{1}=1}^{n} \cdots \sum_{j_{i}=1}^{n} \mathrm{~V}\left(K, n-i ;\left[0, z_{j_{1}}\right], \ldots,\left[0, z_{j_{i}}\right]\right) \\
& =\sum_{J \in\left(\begin{array}{c}
{[n]} \\
i
\end{array}\right)} i ! \mathrm{V}\left(K, n-i ;\left[0, z_{j}\right], j \in J\right),
\end{aligned}
$$

and by Equation (A.41) in [8, App. A.5] it holds

$$
\left(\begin{array}{c}
n \\
i
\end{array}\right) i ! \mathrm{V}\left(K, n-i ;\left[0, z_{j}\right], j \in J\right)=\operatorname{vol}_{n-i}\left(K \mid L \frac{\perp}{J}\right) \operatorname{vol}_{i}\left(P_{J}\right),
$$

for any $J \in\left(\begin{array}{c}{[n]} \\ i\end{array}\right)$. Finally, combining (2.1), (2.2) and (2.3) gives the desired result.

The equality characterization is inherited from Lemma 2.1 since (2.1) is the only step where there could be an inequality.

For later reference, we state the following lemma that can be found for example in [16, Thm. 1] and [5, Lem. 3.1].

Lemma 2.2. Let $K \in \mathcal{K}_{0}^{n}$ and let $L$ be an $i$-dimensional linear subspace of $\mathbb{R}^{n}$. Then

$$
\operatorname{vol}(K) \leq \operatorname{vol}_{n-i}\left(K \mid L^{\perp}\right) \operatorname{vol}_{i}(K \cap L) \leq\left(\begin{array}{c}
n \\
i
\end{array}\right) \operatorname{vol}(K),
$$

and both inequalities are best possible.

Proof of Theorem 1.1. By assumption, we find $n$ linearly independent lattice points $z_{1}, \ldots, z_{n}$ inside $K$. Applying Lemma 1.1 with respect to the lattice parallelepiped $P=\sum_{j=1}^{n}\left[0, z_{j}\right]$ gives

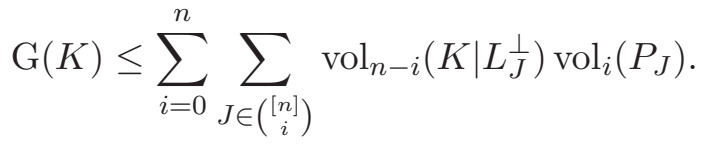


By the construction of the subspaces $L_{J}$, we have

$$
\operatorname{vol}_{i}\left(K \cap L_{J}\right) \geq \operatorname{vol}_{i}\left(\operatorname{conv}\left\{ \pm z_{j}: j \in J\right\}\right)=\frac{2^{i}}{i !} \operatorname{vol}_{i}\left(P_{J}\right)
$$

and together with Lemma 2.2 we get

$$
\begin{aligned}
\mathrm{G}(K) & \leq \sum_{i=0}^{n} \sum_{J \in\left(\begin{array}{c}
{[n]} \\
i
\end{array}\right)} \frac{i !}{2^{i}} \operatorname{vol}_{n-i}\left(K \mid L_{J}^{\perp}\right) \operatorname{vol}_{i}\left(K \cap L_{J}\right) \\
& \leq \operatorname{vol}(K) \sum_{i=0}^{n}\left(\begin{array}{c}
n \\
i
\end{array}\right)^{2} \frac{i !}{2^{i}}=\operatorname{vol}(K) \frac{n !}{2^{n}} L_{n}(2),
\end{aligned}
$$

where $L_{n}(x)=\sum_{k=0}^{n}\left(\begin{array}{c}n \\ k\end{array}\right) \frac{x^{k}}{k !}$ denotes the $n$th Laguerre polynomial. For two functions $f, g: \mathbb{N} \rightarrow \mathbb{R}$, we denote by $f(n) \approx g(n)$ that $\lim _{n \rightarrow \infty} \frac{f(n)}{g(n)}=1$. In Szegö's book [20, p. 199] one finds the approximation

$$
L_{n}(x) \approx \frac{n^{-\frac{1}{4}}}{2 \sqrt{\pi}} \frac{e^{-\frac{x}{2}}}{x^{\frac{1}{4}}} e^{2 \sqrt{x\left(n+\frac{1}{2}\right)}} \quad \text { for all fixed } \quad x>0 .
$$

Therefore, by $\lim _{n \rightarrow \infty} e^{\frac{2 \sqrt{2 n+1}}{n}}=1$, we have

$$
\frac{L_{n}(2)}{2^{n}} \approx \frac{1}{2 e \sqrt{\pi} \sqrt[4]{2 n}} \frac{e^{2 \sqrt{2 n+1}}}{2^{n}}<\frac{e^{2 \sqrt{2 n+1}}}{2^{n}} \leq \frac{1}{(2-\varepsilon)^{n}}
$$

for every $\varepsilon \in(0,1]$ and large enough $n \in \mathbb{N}$. Hence, for large enough $n$, we arrive at

$$
\mathrm{G}(K) \leq \operatorname{vol}(K) \frac{n !}{2^{n}} L_{n}(2) \leq \operatorname{vol}(K) \frac{n !}{(2-\varepsilon)^{n}} .
$$

In order to see that this inequality is asymptotically sharp, we consider the crosspolytope $C_{n, l}^{\star}=\operatorname{conv}\left\{ \pm l e_{1}, \pm e_{2}, \ldots, \pm e_{n}\right\}$. We have $\mathrm{G}\left(C_{n, l}^{\star}\right)=$ $2(n+l)-1$ and $\operatorname{vol}\left(C_{n, l}^{\star}\right)=\frac{2^{n}}{n !} l$. Therefore, $\sqrt[n]{\frac{n ! \operatorname{vol}\left(C_{n, l}^{\star}\right)}{\mathrm{G}\left(C_{n, l}^{\star}\right)}}=\sqrt[n]{\frac{2^{n} l}{2(n+l)-1}}$ tends to 2 when $l$ and $n$ tend to infinity. On the other hand, the above inequality shows that for every $\varepsilon \in(0,1]$ we have $\sqrt[n]{\frac{n ! \operatorname{vol}(K)}{\mathrm{G}(K)}} \geq 2-\varepsilon$ for $n \rightarrow \infty$.

\section{An application of Theorem 1.1}

In this section, we give the details of the proof of Theorem 1.2. We proceed by showing that Theorem 1.1 can be used to derive an improvement of

$$
\mathrm{G}(K) \operatorname{vol}\left(K^{\star}\right) \leq 6^{n} .
$$

This inequality is due to Gillet and Soulé [9] and holds for every $K \in \mathcal{K}_{0}^{n}$ with $\operatorname{dim}\left(K \cap \mathbb{Z}^{n}\right)=n$. The volume of the Euclidean unit ball $B_{n}$ is denoted by

$$
\kappa_{n}=\operatorname{vol}\left(B_{n}\right)=\frac{\pi^{\frac{n}{2}}}{\Gamma\left(\frac{n}{2}+1\right)},
$$

where $\Gamma(z)=\int_{0}^{\infty} e^{-t} t^{z-1} d t$ is the gamma function (cf. [8, p. 13]). 
Corollary 3.1. For every $\varepsilon>0$ there exists an $n(\varepsilon) \in \mathbb{N}$ such that for every $n \geq n(\varepsilon)$ and every $K \in \mathcal{K}_{0}^{n}$ with $\operatorname{dim}\left(K \cap \mathbb{Z}^{n}\right)=n$, we have

$$
\frac{c^{n}}{n !} \leq \mathrm{G}(K) \operatorname{vol}\left(K^{\star}\right) \leq(\pi+\varepsilon)^{n} .
$$

Here, $c \leq 2$ is an absolute constant and the lower bound holds for every $n \in \mathbb{N}$ and arbitrary $K \in \mathcal{K}_{0}^{n}$.

Proof. For the lower bound we combine Inequality (1.1) and the estimate

$$
\operatorname{vol}(K) \operatorname{vol}\left(K^{\star}\right) \geq \frac{C^{n}}{n !}
$$

which is due to Bourgain and Milman [5] and holds for some universal constant $C \leq 4$. Indeed, we have

$$
\mathrm{G}(K) \operatorname{vol}\left(K^{\star}\right) \geq \frac{1}{2^{n}} \operatorname{vol}(K) \operatorname{vol}\left(K^{\star}\right) \geq \frac{(C / 2)^{n}}{n !} .
$$

Now we restrict to $K \in \mathcal{K}_{0}^{n}$ with $\operatorname{dim}\left(K \cap \mathbb{Z}^{n}\right)=n$. The exact inequality (2.4) in the proof of Theorem 1.1 and the Blaschke-Santaló [17] inequality imply that

$$
\mathrm{G}(K) \operatorname{vol}\left(K^{\star}\right) \leq \frac{n ! L_{n}(2)}{2^{n}} \operatorname{vol}(K) \operatorname{vol}\left(K^{\star}\right) \leq \frac{n ! \kappa_{n}^{2} L_{n}(2)}{2^{n}} .
$$

Hence, by (2.5), we have that for every $\varepsilon^{\prime} \in(0,1]$ and large enough $n \in \mathbb{N}$

$$
\mathrm{G}(K) \operatorname{vol}\left(K^{\star}\right) \leq \frac{n ! \kappa_{n}^{2}}{\left(2-\varepsilon^{\prime}\right)^{n}}
$$

Stirling approximation and $\kappa_{n}=\frac{\pi^{\frac{n}{2}}}{\Gamma\left(\frac{n}{2}+1\right)}$ give

$$
\frac{n ! \kappa_{n}^{2}}{\left(2-\varepsilon^{\prime}\right)^{n}} \approx \frac{\sqrt{2 \pi n}\left(\frac{n}{e}\right)^{n} \pi^{n}}{\left(2-\varepsilon^{\prime}\right)^{n} \Gamma\left(\frac{n}{2}+1\right)^{2}} \approx \frac{\sqrt{2 \pi n}\left(\frac{n}{e}\right)^{n} \pi^{n}}{\left(2-\varepsilon^{\prime}\right)^{n} \pi n\left(\frac{n}{2 e}\right)^{n}} \leq\left(\frac{2 \pi}{2-\varepsilon^{\prime}}\right)^{n} .
$$

For every $\varepsilon \in(0,1)$ there exists an $\varepsilon^{\prime} \in(0,1)$ such that $\frac{2 \pi}{2-\varepsilon^{\prime}} \leq \pi+\varepsilon$, and we conclude that for large $n$, the inequality $\mathrm{G}(K) \operatorname{vol}\left(K^{\star}\right) \leq(\pi+\varepsilon)^{n}$ holds.

In the planar case, we are able to give sharp bounds. Two sets are called unimodularly equivalent if there is a lattice preserving affine transformation that maps one onto the other. With $e_{i}$ we denote the $i$ th unit vector in $\mathbb{R}^{n}$.

Proposition 3.1. Let $K \in \mathcal{K}_{0}^{2}$ be such that $\operatorname{dim}\left(K \cap \mathbb{Z}^{2}\right)=2$. Then

$$
2 \leq \mathrm{G}(K) \operatorname{vol}\left(K^{\star}\right) \leq 21 .
$$

In the upper bound, equality holds if and only if $K$ is unimodularly equivalent to the hexagon $H=\operatorname{conv}\left\{ \pm e_{1}, \pm e_{2}, \pm\left(e_{1}+e_{2}\right)\right\}$. The lower bound is also best possible and holds for arbitrary $K \in \mathcal{K}_{0}^{2}$. 
Proof. For the upper bound we can restrict to lattice polygons $P \in \mathcal{P}_{0}^{2}$ since for $P_{K}=\operatorname{conv}\left\{K \cap \mathbb{Z}^{2}\right\}$ we clearly have $\mathrm{G}(K) \operatorname{vol}\left(K^{\star}\right) \leq \mathrm{G}\left(P_{K}\right) \operatorname{vol}\left(P_{K}^{\star}\right)$. By the well-known formula of Pick [14] we get

$$
\begin{aligned}
\mathrm{G}(P) \operatorname{vol}\left(P^{\star}\right) & =\left(\operatorname{vol}(P)+\frac{1}{2} \mathrm{G}(\partial P)+1\right) \operatorname{vol}\left(P^{\star}\right) \\
& =\left(\operatorname{vol}(P)+\frac{1}{2} \mathrm{G}(P)-\frac{1}{2} \mathrm{G}(\operatorname{int} P)+1\right) \operatorname{vol}\left(P^{\star}\right)
\end{aligned}
$$

and therefore

$$
\mathrm{G}(P) \operatorname{vol}\left(P^{\star}\right)=2 \operatorname{vol}(P) \operatorname{vol}\left(P^{\star}\right)+\operatorname{vol}\left(P^{\star}\right)(2-\mathrm{G}(\text { int } P)) .
$$

Using the Blaschke-Santaló inequality [17] in the plane gives $\mathrm{G}(P) \operatorname{vol}\left(P^{\star}\right) \leq$ $2 \operatorname{vol}(P) \operatorname{vol}\left(P^{\star}\right) \leq 2 \pi^{2}<21$, whenever $\mathrm{G}($ int $P)>1$. Up to unimodular equivalence there are only three centrally symmetric lattice polygons with exactly one interior lattice point: the square $[-1,1]^{2}$, the diamond $\operatorname{conv}\left\{ \pm e_{1}, \pm e_{2}\right\}$ and the hexagon $H$ (see for example [13, Prop. 2.1]). Among these three the hexagon is the only maximizer of $\mathrm{G}(P) \operatorname{vol}\left(P^{\star}\right)$.

For the lower bound we use Mahler's inequality $\operatorname{vol}(K) \operatorname{vol}\left(K^{\star}\right) \geq 8$, for $K \in \mathcal{K}_{0}^{2}$ (see [12]). By the same lines as in the proof of Corollary 3.1 this yields $\mathrm{G}(K) \operatorname{vol}\left(K^{\star}\right) \geq 2$ which is best possible as shown by the squares $[-1+\varepsilon, 1-\varepsilon]^{2}$, for small $\varepsilon>0$.

Remark 3.1. Based on computer experiments in small dimensions, we conjecture that the hexagon in the plane is an exception and that for $n \geq 3$ the maximizing example is the standard crosspolytope $C_{n}^{\star}=\operatorname{conv}\left\{ \pm e_{1}, \ldots, \pm e_{n}\right\}$, i.e. $\mathrm{G}(K) \operatorname{vol}\left(K^{\star}\right) \leq(2 n+1) 2^{n}$ for every $K \in \mathcal{K}_{0}^{n}$ with $\operatorname{dim}\left(K \cap \mathbb{Z}^{n}\right)=n$.

Finally we are ready to prove Theorem 1.2. The arguments are similar to those given by Gillet and Soulé [9, Sect. 1.6]. We repeat them here with the necessary adjustments to our bounds and for the sake of completeness.

Proof of Theorem 1.2. The upper bound can be derived by applying the lower bound to $K^{\star}$ and by using Inequality (3.1) of Bourgain and Milman. In fact, there is an absolute constant $c \leq 4$ with

$$
\frac{\mathrm{G}(K)}{\mathrm{G}\left(K^{\star}\right) \operatorname{vol}(K)}=\frac{\mathrm{G}(K) \operatorname{vol}\left(K^{\star}\right)}{\mathrm{G}\left(K^{\star}\right) \operatorname{vol}(K) \operatorname{vol}\left(K^{\star}\right)} \leq \frac{(\pi+\varepsilon)^{n}}{\operatorname{vol}(K) \operatorname{vol}\left(K^{\star}\right)} \leq \frac{(\pi+\varepsilon)^{n} n !}{c^{n}} .
$$

Here, $\varepsilon>0$ and $n \in \mathbb{N}$ is large enough.

For the lower bound, let $L=\operatorname{lin}\left(K^{\star} \cap \mathbb{Z}^{n}\right)$ and $k=\operatorname{dim} L$. Let us abbreviate $L_{n}=L_{n}(2)$. We use the exact inequality (3.2) from the proof of Corollary 3.1 in the sublattice $\mathbb{Z}^{n} \cap L$ and obtain

$$
\begin{aligned}
\mathrm{G}\left(K^{\star}\right) & =\mathrm{G}\left(K^{\star} \cap L\right) \leq \frac{k ! \kappa_{k}^{2} L_{k}}{2^{k}} \frac{\operatorname{det}\left(\left(\mathbb{Z}^{n} \cap L\right)^{\star}\right)}{\operatorname{vol}_{k}\left(\left(K^{\star} \cap L\right)^{\star}\right)} \\
& =\frac{k ! \kappa_{k}^{2} L_{k}}{2^{k}} \frac{\operatorname{det}\left(\mathbb{Z}^{n} \mid L\right)}{\operatorname{vol}_{k}(K \mid L)} .
\end{aligned}
$$


By $K=\left(K^{\star}\right)^{\star}$, we have int $K=\left\{x \in K:\left|x^{\top} y\right|<1, \forall y \in K^{\star}\right\}$ and thus $x^{\top} y=0$ for all $x \in \operatorname{int} K \cap \mathbb{Z}^{n}$ and $y \in K^{\star} \cap \mathbb{Z}^{n}$. Therefore, using Theorem 1.1 in the sublattice $\mathbb{Z}^{n} \cap L^{\perp}$, we get

$$
\begin{aligned}
\mathrm{G}(K) & \geq \mathrm{G}(\operatorname{int} K)=\mathrm{G}\left(\operatorname{int} K \cap L^{\perp}\right) \\
& \geq \frac{1}{2^{n-k}} \frac{\operatorname{vol}_{n-k}\left(\operatorname{int} K \cap L^{\perp}\right)}{\operatorname{det}\left(\mathbb{Z}^{n} \cap L^{\perp}\right)}=\frac{1}{2^{n-k}} \frac{\operatorname{vol}_{n-k}\left(K \cap L^{\perp}\right)}{\operatorname{det}\left(\mathbb{Z}^{n} \cap L^{\perp}\right)} .
\end{aligned}
$$

Combining (3.3), (3.4) and the lower bound in Lemma 2.2 gives

$$
\frac{\mathrm{G}(K)}{\mathrm{G}\left(K^{\star}\right)} \geq \frac{4^{k}}{2^{n} k ! \kappa_{k}^{2} L_{k}} \frac{\operatorname{vol}_{n-k}\left(K \cap L^{\perp}\right) \operatorname{vol}_{k}(K \mid L)}{\operatorname{det}\left(\mathbb{Z}^{n} \cap L^{\perp}\right) \operatorname{det}\left(\mathbb{Z}^{n} \mid L\right)} \geq \frac{4^{k} \operatorname{vol}(K)}{2^{n} k ! \kappa_{k}^{2} L_{k}} .
$$

What remains is to show that the function $g(k)=\frac{4^{k}}{k ! \kappa_{k}^{2} L_{k}}$ is nonincreasing for $k \geq 0$, because together with the previous inequality we then arrive at

$$
\frac{\mathrm{G}(K)}{\mathrm{G}\left(K^{\star}\right) \operatorname{vol}(K)} \geq \frac{4^{k}}{2^{n} k ! \kappa_{k}^{2} L_{k}} \geq \frac{2^{n}}{n ! \kappa_{n}^{2} L_{n}} .
$$

In the proof of Corollary 3.1, we have seen that for every $\varepsilon>0$ the right hand side of the above inequality is at least $(\pi+\varepsilon)^{-n}$ for large enough $n \in \mathbb{N}$.

Now $g(k) \geq g(k+1)$ if and only if $\frac{k+1}{4} \geq \frac{\kappa_{k}^{2}}{\kappa_{k+1}^{2}} \frac{L_{k}}{L_{k+1}}$. By the estimate $\frac{\kappa_{k}^{2}}{\kappa_{k+1}^{2}} \leq \frac{k+2}{2 \pi}$ (see [2, Lem. 1]), it is therefore enough to prove $\frac{k+1}{4} \geq \frac{k+2}{2 \pi} \frac{L_{k}}{L_{k+1}}$. After an elementary calculation, we see that this follows from the recurrence relation $L_{k+1}=\sum_{i=0}^{k+1}\left(\begin{array}{c}k+1 \\ i\end{array}\right) \frac{2^{i}}{i !}=2 L_{k}-\frac{2^{k}(k-1)}{(k+1) !}$.

Acknowledgment. We thank Martin Henk for many valuable comments and suggestions.

\section{REFERENCES}

[1] U. Betke, J. M. Wills, Stetige und diskrete Funktionale konvexer Körper, Contributions to geometry (Proc. Geom. Sympos., Siegen, 1978), Birkhäuser, Basel-Boston, Mass., (1979), 226-237.

[2] U. Betke, P. Gritzmann, J. M. Wills, Slices of L. Fejes Tóth's sausage conjecture, Mathematika 29 (1982), 194-201.

[3] C. Bey, M. Henk, J. M. Wills, Notes on the roots of Ehrhart polynomials, Discrete Comput. Geom. 38 (2007), no. 1, 81-98.

[4] H. F. Blichfeldt, Notes on Geometry of Numbers in The october meeting of the San Francisco section, Bull. Amer. Math. Soc. 27 (1921), no. 4, 150-153.

[5] J. Bourgain, V. D. Milman, New volume ratio properties for convex symmetric bodies in $\mathbb{R}^{n}$, Invent. Math. 88 (1987), 319-340.

[6] J. G. van der Corput, Verallgemeinerung einer Mordellschen Beweismethode in der Geometrie der Zahlen II, Acta Arith. 2 (1936), 145-146.

[7] H. Davenport, On a principle of Lipschitz, J. London Math. Soc. 26 (1951), 179-183.

[8] R. J. Gardner, Geometric Tomography, Cambridge University Press, New York, 1995.

[9] H. Gillet, C. Soulé, On the number of lattice points in convex symmetric bodies and their duals, Isr. J. Math. 74 (1991), no. 2-3, 347-357.

[10] P. Gritzmann, J. M. Wills, Lattice points, Handbook of convex geometry (P. M. Gruber and J. M. Wills, eds.), vol. B, North-Holland, Amsterdam, 1993. 
[11] M. Henk, M. Henze, J. M. Wills, Blichfeldt-type inequalities and central symmetry, Adv. Geom. 11 (2011), no. 4, 731-744.

[12] K. Mahler, Ein Minimalproblem für konvexe Polygone, Mathematica (Zutphen) B7 (1938), 118-127.

[13] B. Nill, Gorenstein toric Fano varieties, Manuscripta Math. 116 (2005), 183-210.

[14] G. A. Pick, Geometrisches zur Zahlenlehre, Sitzungsber. Lotus Prag 19 (1899), 311319.

[15] O. Pikhurko, Lattice points in lattice polytopes, Mathematika 48 (2001), 15-24.

[16] C. A. Rogers, G. C. Shephard, Convex bodies associated with a given convex body, J. London Math. Soc. 33 (1958), 270-281.

[17] L. A. Santaló, Un invariante afin para los cuerpos convexos del espacio de $n$ dimensiones, Portugal. Math. 8 (1949), 155-161.

[18] R. Schneider, Convex bodies: the Brunn-Minkowski theory, Cambridge University Press, Cambridge, 1993.

[19] E. Schulte, Tilings, Handbook of convex geometry (P. M. Gruber and J. M. Wills, eds.), vol. B, North-Holland, Amsterdam, 1993.

[20] G. Szegö, Orthogonal polynomials, 4th edition, Amer. Math. Soc. Colloq. Publ., vol. 23, Amer. Math. Soc., Providence, RI, 1975.

FAkultät FÜr Mathematik, Otto-von-Guericke Universität Magdeburg, UniVersitätsplatz 2, D-39106 Magdeburg, Germany

E-mail address: matthias.henze@ovgu.de 